\title{
Field-Theoretical Methods and Nonextensive Systems
}

\author{
Renio S. Mendes \\ Departamento de Física, \\ Universidade Estadual de Maringá, \\ Av. Colombo 5790, 87020-900 Maringá-PR, Brazil \\ Received 07 December, 1998
}

\begin{abstract}
Traditional field-theoretical methods to study extensive many-particle systems are generalized to discuss nonextensive situations. In particular, generalizations of Green functions, path integral, and Gaussian integration are performed in the context of nonextensive Tsallis statistical mechanics. These developments employ integral representations that connect the usual and the generalized cases.
\end{abstract}

\section{Introduction}

The Boltzmann-Gibbs statistical mechanics and the standard thermodynamics are very useful in the discussion of extensive systems. However $\Gamma$ they are not good to study situations where nonextensive effects occur $\Gamma$ for instance $\Gamma$ systems involving long-range inter-

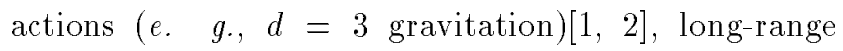
microscopic memory[3] $\Gamma$ and systems with a relevant (multi)fractal-like structure. On the other hand $\mathrm{T}$ the investigations based on the Tsallis entropy[4] mainly focuses on the study of nonextensive problems. Examples of these applications are the Lévy superdiffusion[5Г 6$]$ and anomalous correlated diffusion[7] $\Gamma$ turbulence in a two-dimensional pure electron plasma[8] $\Gamma$ thermalization of an electron-phonon system[9] $\Gamma$ astrophysical applications[10] Tdynamical systems[11 112$]$ Tamong others[13].

It is important to remark that the Tsallis entropy extends the Boltzmann-Gibbs (BG) oneTbecause it depends on a real parameter $q$ and it is reduced to the BG one in the limit $q \rightarrow 1$. Consequently it is natural to investigate how the formalism based on the BG entropy can be enlarged in order to incorporate the Tsallis one $\Gamma$ $i$. $e$. in order to contain the case $q \neq 1$. In this direction Tit was verified that the Legendre structure[14T15] is preserved $\Gamma$ dynamic linear response theory[16] $\Gamma$ perturbation and variational methods for calculation of thermodynamic quantities[17Г18] Tand Green functions can be generalized[19Г20Г21] Tamong others[13].

This work is dedicated to discuss generalizations that employ field-theoretical methods. More precisely $\Gamma$ generalizations of the Green functions that incorporate the nonextensive Tsallis statistical mechanics are focused here as well as the corresponding path integral formulation. Furthermore $\Gamma$ a generalization of the Gaussian integrals based also on the Tsallis statistics is analyzed. In order to naturally relate a generalized case with the usual one $\Gamma$ integral representations are employed. Before starting the discussion about field theoretical methods applied to the generalized Tsallis statistical mechanicsTa brief review about Tsallis statisticsTincluding integral representations $\Gamma$ is presented in Sec. II in order to mainly establish the notation employed in this work. By using integral representations $\Gamma$ the generalized Green functions are discussed in Sec. III. The Hartree and Hartree-Fock approximations for the generalized Green functions are considered in Sec. IV. The path integral formulation for Tsallis statistics is presented in Sec. V. In the context of the normalized version of the Tsallis statistical mechanicsTGreen functions are discussed in Sec. VI. The generalization of the Gaussian integrals is the main focus of Sec. VII. Sec. VIII contains a summary and concluding remarks. 


\section{Tsallis statistics and integral representations}

The nonextensive entropy (Tsallis entropy)[4]

$$
S_{q}=k \operatorname{Tr} \frac{\hat{\rho}-\hat{\rho}^{q}}{q-1}
$$

is a fundamental object in the discussion of the so called generalized statistical mechanics. In Eq. (1) $\hat{\rho}$ is the density matrix $\Gamma q \in \mathbf{R}$ characterizes the degree of nonextensivity $\Gamma$ and $k$ is a positive constant. In order to obtain the statistical weight $\Gamma$ the entropy (1) is maximized subject to some constraints. In the grand-canonical ensemble $\Gamma$ for instance $\Gamma$ the constraints are chosen as[4Г14]

$$
\begin{gathered}
\operatorname{Tr} \hat{\rho}=1, \\
\operatorname{Tr} \hat{\rho}^{q} \hat{H}=U_{q},
\end{gathered}
$$

and

$$
\operatorname{Tr} \hat{\rho}^{q} \hat{N}=N_{q}
$$

where $\hat{H}$ and $\hat{N}$ are respectively the Hamiltonian and number operators. In this wayT the generalized grandcanonical statistical matrix becomes

$$
\hat{\rho}=[1-(1-q) \beta(\hat{H}-\mu \hat{N})]^{1 /(1-q)} / Z_{q}
$$

with the generalized grand-partition function defined as

$$
Z_{q}=\operatorname{Tr}[1-(1-q) \beta(\hat{H}-\mu \hat{N})]^{1 /(1-q)}
$$

Thus the probabilities in the grand-canonical ensemble becomes

$$
p_{n}=\left[1-(1-q) \beta\left(E_{n}-\mu N_{n}\right)\right]^{1 /(1-q)} / Z_{q},
$$

where it was supposed that $[\hat{H}, \hat{N}]_{-}=0$. In Eq. (7) it is also assumed that $1-(1-q) \beta\left(E_{n}-\mu N_{n}\right) \geq 0$. When this condition is not satisfied $\Gamma p_{n}=0$ must be used in order to retain the probabilistic interpretation for $p_{n}$. In this way there is a cut off when $1-(1-q) \beta\left(E_{n}-\mu N_{n}\right)<0$. The Lagrange multipliers $\beta$ and $\mu$ in the above equations are considered respectively as the inverse of the generalized temperature $\Gamma 1 /(k T)$ Tand generalized chemical potential. Furthermore $\Gamma$

$$
\langle A\rangle_{q} \equiv \operatorname{Tr} \hat{\rho}^{q} \hat{A}
$$

is usually referred as the $q$ expectation value of $\hat{A}$. Note that the above equations reduce to the usual ones in the limit $q \rightarrow 1$. Thus $[1-(1-q) x]^{1 /(1-q)}$ can be considered a generalization of $\exp (-x)$. A normalized definition of generalized mean values that also reduces to the usual in the limit $q \rightarrow 1$ is discussed in Sec. VI.

Powerful tools to calculate the generalized partition functionsTand consequently other important statistical quantities $\Gamma$ are the integral representations. By using an integral representation $\Gamma Z_{q}$ can be written in terms of the usual partition function $\Gamma Z_{1}$. In fact $\Gamma$ the contour integral[22]

$$
b^{1-z} \frac{i}{2 \pi} \int_{C} \mathrm{~d} u(-u)^{-z} \exp (-b u)=\frac{1}{\Gamma(z)},
$$

with $b=1-(1-q) \beta(\hat{H}-\mu \hat{N})$ and $z=1+1 /(1-q)$ leads to

$$
Z_{q}(\beta, \mu)=\int_{C} \mathrm{~d} u K_{q}^{(1)} Z_{1}(-\beta u(1-q), \mu)
$$

where

$$
K_{q}^{(1)}=i \frac{\Gamma(1 /(1-q))}{2 \pi(q-1) u}(-u)^{-1 /(1-q)} \exp (-u) .
$$

In Eq. (9) $b>0$ and Re $z>0 \Gamma$ where the contour $C$ starts from $+\infty$ on the real axisTencircles the origin once counterclockwise $\Gamma$ and returns to $+\infty$. This representation is very general from which others can be obtained. For instance The Hilhorst representation (private communication to Tsallis)[23] is based on the Euler definition of the Gamma function $\Gamma$

$$
b^{z} \int_{0}^{\infty} \mathrm{d} x x^{z-1} \exp (-b x)=\Gamma(z) \quad(\operatorname{Re} b>0 \text { and } \operatorname{Re} z>0),
$$

and the Lenzi representation[24] employs the integral identity[25]

$$
\frac{b^{1-z} \exp (a b)}{2 \pi} \int_{-\infty}^{\infty} \mathrm{d} t \frac{\exp (i b t)}{(a+i t)^{z}}=\frac{1}{\Gamma(z)} \text { for } b>0,
$$




$$
=0 \text { for } b<0 \text {, }
$$

where $a>0$ TRe $z>0$ Tand $-\pi / 2<\arg (a+i t)<\pi / 2$. The Hilhorst representation is usually useful in the $q>1$ case and it was applied to study a set of particles without interaction[23]. On the other handT the Lenzi representation is usually applicable to $q<1$ and accomplishes the cut off condition previously discussed. It was employed to obtain the exact solution of the blackbody radiation[26] and to establish the perturbative and variational methods for quantum systems in the context of Tsallis statistical mechanics[18]. The first discussion about integral representation for $q<1$ was presented by Prato[27] and it was employed to study the classical ideal gas.

$$
\hat{N}(t)=\int \mathrm{d}^{d} \mathbf{x} \hat{n}(\mathbf{x}, t) \quad\left(\hat{n}(\mathbf{x}, t)=\hat{\psi}^{\dagger}(\mathbf{x}, t) \hat{\psi}(\mathbf{x}, t)\right)
$$

and the Hamiltonian becomes

$$
\begin{aligned}
\hat{H}(t) & =\int \mathrm{d}^{d} \mathbf{x} \frac{\nabla \hat{\psi}^{\dagger}(\mathbf{x}, t) \cdot \nabla \hat{\psi}(\mathbf{x}, t)}{2 m} \\
& +\int \mathrm{d}^{d} \mathbf{x} \mathrm{d}^{d} \mathbf{y} \hat{\psi}^{\dagger}(\mathbf{x}, t) \hat{\psi}^{\dagger}(\mathbf{y}, t) V(\mathbf{x}-\mathbf{y}) \hat{\psi}(\mathbf{y}, t) \hat{\psi}(\mathbf{x}, t)
\end{aligned}
$$

It was employed $\hbar=1 \Gamma m$ and $d$ to represent respectively the particle mass and the spatial dimension $\Gamma$ and for simplicity a two-body potential $V(\mathbf{x}-\mathbf{y})$.

The creation and annihilation operators obey the equal time (anti) commutation relation $\left([\hat{A}, \hat{B}]_{\mp}=\hat{A} \hat{B} \mp \hat{A} \hat{B}\right) \Gamma$

$$
\left[\hat{\psi}^{\dagger}(\mathbf{x}, t), \hat{\psi}^{\dagger}(\mathbf{y}, t)\right]_{\mp}=[\hat{\psi}(\mathbf{x}, t), \hat{\psi}(\mathbf{y}, t)]_{\mp}=0
$$

and

$$
\left[\hat{\psi}(\mathbf{x}, t), \hat{\psi}^{\dagger}(\mathbf{y}, t)\right]_{\mp}=\delta(\mathbf{x}-\mathbf{y})
$$

In these last two expressions The upper sign $(-)$ refers to Bose-Einstein particles and the lower sign $(+)$ refers to Fermi-Dirac particles. Furthermore $\Gamma$ the dynamics of any operator $\hat{\mathcal{O}}(t)$ is dictated by the Heisenberg equation $\Gamma$

$$
i \frac{\mathrm{d} \hat{\mathcal{O}}(t)}{\mathrm{d} t}=[\hat{\mathcal{O}}(t), \hat{H}(t)]_{-}
$$

Thus $\hat{H}(t)$ and $\hat{N}(t)$ are time independent because $[\hat{H}(t), \hat{H}(t)]_{-}=0$ and $[\hat{N}(t), \hat{H}(t)]_{-}=0$. 
In the usual statistical mechanics the expectation value of any operator $\hat{\mathcal{O}}(t)$ Twhen the grand-canonical ensemble is employedTis given by

$$
\langle\hat{\mathcal{O}}\rangle_{1}=\frac{\operatorname{Tr} \exp (-\beta(\hat{H}-\mu \hat{N})) \hat{\mathcal{O}}}{\operatorname{Tr} \exp (-\beta(\hat{H}-\mu \hat{N}))}
$$

By using this notation Tthe $n$-particle Green function is defined by

$$
\begin{aligned}
& G_{1}^{(n)}\left(\mathbf{x}_{1}, t_{1}, \ldots, \mathbf{x}_{n}, t_{n}, \mathbf{y}_{1}, t_{1}^{\prime}, \ldots, \mathbf{y}_{n}, t_{n}^{\prime} ; \beta, \mu\right)= \\
& \frac{1}{i^{n}}\left\langle\mathrm{~T}\left(\hat{\psi}\left(\mathbf{x}_{1}, t_{1}\right) \ldots \hat{\psi}\left(\mathbf{x}_{n}, t_{n}\right) \hat{\psi}^{\dagger}\left(\mathbf{y}_{1}, t_{1}^{\prime}\right) \ldots \hat{\psi}^{\dagger}\left(\mathbf{y}_{n}, t_{n}^{\prime}\right)\right)\right\rangle_{1} .
\end{aligned}
$$

The symbol T represents the Wick time-ordering operationTand arranges the product of operators in chronological orderTfor instance $\Gamma$

$$
\begin{aligned}
& \mathrm{T}\left(\hat{\psi}(\mathbf{x}, t) \hat{\psi}^{\dagger}\left(\mathbf{y}, t^{\prime}\right)\right)=\quad \hat{\psi}(\mathbf{x}, t) \hat{\psi}^{\dagger}\left(\mathbf{y}, t^{\prime}\right) \quad \text { for } \quad t>t^{\prime} \\
& \pm \hat{\psi}^{\dagger}\left(\mathbf{y}, t^{\prime}\right) \hat{\psi}(\mathbf{x}, t) \text { for } t<t^{\prime}
\end{aligned}
$$

The sign $(+)$ is used for bosons $\Gamma$ when the order between $\psi$ and $\psi^{\dagger}$ is changed. On the other hand $\mathrm{the}$ sign $(-)$ is used for fermions. The upper sign will be employed for bosons and the lower sign is used for fermions in the following discussions.

In connection with one-particle Green functionTit is employed the correlation functions

$$
G_{1>}\left(\mathbf{x}, t, \mathbf{y}, t^{\prime} ; \beta, \mu\right)=\frac{1}{i}\left\langle\hat{\psi}(\mathbf{x}, t) \hat{\psi}^{\dagger}\left(\mathbf{y}, t^{\prime}\right)\right\rangle_{1}
$$

and

$$
G_{1<}\left(\mathbf{x}, t, \mathbf{y}, t^{\prime} ; \beta, \mu\right)=\frac{ \pm}{i}\left\langle\hat{\psi}^{\dagger}\left(\mathbf{y}, t^{\prime}\right) \hat{\psi}(\mathbf{x}, t)\right\rangle_{1} .
$$

Thus $\Gamma G_{1}^{(1)}=G_{1}$ for $t>t^{\prime}$ and $G_{1}^{(1)}=G_{1<}$ for $t<t^{\prime}$. Because of the time and spatial translational invariance of the Hamiltonian $(15) \Gamma G_{1}^{(1)}$ depends only on $\mathbf{r}=\mathbf{x}-\mathbf{y}$ and $\tilde{t}=t-t^{\prime} \Gamma i$. e. $G_{1}^{(1)}=G_{1}^{(1)}(\mathbf{r}, \tilde{t} ; \beta, \mu)$. Furthermore $\Gamma$ it is introduced the spectral function defined by

$$
\begin{aligned}
A_{1}(\mathbf{y} ; \omega ; \beta, \mu) & =G_{1>}(\mathbf{x}, \mathbf{y} ; \omega ; \beta, \mu) \mp G_{1<}(\mathbf{x}, \mathbf{y} ; \omega ; \beta, \mu) \\
& =\int \frac{\mathrm{d}^{d} \mathbf{p}}{(2 \pi)^{d}} \exp (i \mathbf{p} \cdot \mathbf{r}) A_{1}(\mathbf{p}, \omega ; \beta, \mu) \\
& =\int \frac{\mathrm{d}^{d} \mathbf{p}}{(2 \pi)^{d}} \exp (i \mathbf{p} \cdot \mathbf{r})\left[G_{1>}(\mathbf{p}, \omega ; \beta, \mu) \mp G_{1<}(\mathbf{p}, \omega ; \beta, \mu)\right]
\end{aligned}
$$

where

$$
G_{1>}(\mathbf{p}, \omega ; \beta, \mu)=i \int \mathrm{d}^{d} \mathbf{r} \int_{-\infty}^{\infty} \mathrm{d} \tilde{t} \exp (-i \mathbf{p} \cdot \mathbf{r}+i \omega \tilde{t}) G_{1>}(\mathbf{r}, \tilde{t} ; \beta, \mu)
$$

and

$$
G_{1<}(\mathbf{p}, \omega ; \beta, \mu)= \pm i \int \mathrm{d}^{d} \mathbf{r} \int_{-\infty}^{\infty} \mathrm{d} \tilde{t} \exp (-i \mathbf{p} \cdot \mathbf{r}+i \omega \tilde{t}) G_{1<}(\mathbf{r}, \tilde{t} ; \beta, \mu)
$$

From these definitions and Eq. (17) the sum rule follows

$$
\begin{aligned}
\int \frac{\mathrm{d} \omega}{2 \pi} A_{1}(\mathbf{x}, \mathbf{y} ; \omega ; \beta, \mu) & =\int \frac{\mathrm{d} \omega}{2 \pi} \int \frac{\mathrm{d}^{d} \mathbf{p}}{(2 \pi)^{d}} \exp (i \mathbf{p} \cdot \mathbf{r}) A_{1}(\mathbf{p}, \omega ; \beta, \mu) \\
& =\int \frac{\mathrm{d} \omega}{2 \pi} \int_{-\infty}^{\infty} \mathrm{d} \tilde{t} \exp (i \omega \tilde{t})\left\langle\psi(\mathbf{r}, \tilde{t}) \psi^{\dagger}(\mathbf{0}, 0) \mp \psi^{\dagger}(\mathbf{0}, 0) \psi(\mathbf{r}, \tilde{t})\right\rangle_{1} \\
& =\left\langle\left[\psi(\mathbf{r}, 0), \psi^{\dagger}(\mathbf{0}, 0)\right]_{\mp}\right\rangle_{1}=\delta(\mathbf{x}-\mathbf{y})
\end{aligned}
$$


or alternatively

$$
\int_{\infty}^{\infty} \frac{\mathrm{d} \omega}{2 \pi}\left[G_{1>}(\mathbf{p}, \omega ; \beta, \mu) \mp G_{1<}(\mathbf{p}, \omega ; \beta, \mu)\right]=1 .
$$

It is important to emphasize also that correlation functions satisfy the fundamental relation

$$
\left.G_{1<}\left(\mathbf{x}, t, \mathbf{y}, t^{\prime} ; \beta, \mu\right)\right|_{t=0}= \pm\left. e^{\beta \mu} G_{1>}\left(\mathbf{x}, t, \mathbf{y}, t^{\prime} ; \beta, \mu\right)\right|_{t=-i \beta}
$$

In terms of the spectral function and the Fourier transform of the correlation functionsTthis relation can be written as

$$
G_{1>}(\mathbf{p}, \omega ; \beta, \mu)=[1 \pm f(\omega ; \beta, \mu)] A_{1}(\mathbf{p}, \omega ; \beta, \mu)
$$

and

$$
G_{1<}(\mathbf{p}, \omega ; \beta, \mu)=f(\omega ; \beta, \mu) A_{1}(\mathbf{p}, \omega ; \beta, \mu)
$$

where

$$
f(\omega ; \beta, \mu)=\frac{1}{\exp (\beta(\omega-\mu)) \mp 1} .
$$

Here the function $f(\omega ; \beta, \mu)$ is the average occupation number in the grand-canonical ensemble of a mode with energy $\omega$ Tand the spectral function $A_{1}(\mathbf{p}, \omega ; \beta, \mu)$ is a weighting function with total weight unity (see Eq. (27)). For free particlesTfor instance Tthe weighting function is given by $A_{1}(\mathbf{p}, \omega ; \beta, \mu)=2 \pi \delta\left(\omega-\mathbf{p}^{2} /(2 m)\right)$.

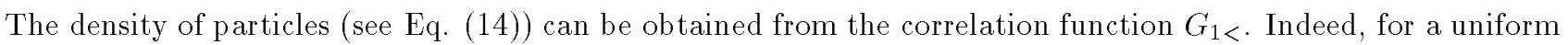
system $\Gamma$

$$
\begin{aligned}
\langle\hat{n}(\mathbf{0}, 0)\rangle_{1} & =\langle\hat{n}(\mathbf{x}, t)\rangle_{1}=\left\langle\hat{\psi}^{\dagger}(\mathbf{x}, t) \hat{\psi}(\mathbf{x}, t)\right\rangle_{1} \\
& = \pm i G_{1<}(\mathbf{x}, t, \mathbf{x}, t ; \beta, \mu)=\int_{-\infty}^{\infty} \frac{\mathrm{d} \omega}{2 \pi} \int \frac{\mathrm{d}^{d} \mathbf{p}}{(2 \pi)^{d}} G_{1<}(\mathbf{p}, \omega ; \beta, \mu) .
\end{aligned}
$$

From $G_{1<}$ the expectation value of the Hamiltonian (15) can be also obtained. To verify that this affirmation is true TfirstTnote that the equation of motion (18) leads to

$$
\left(i \frac{\partial}{\partial t}+\frac{\nabla_{\mathbf{x}}^{2}}{2 m}\right) \hat{\psi}(\mathbf{x}, t)=\int \mathbf{d}^{d} \mathbf{y} V(\mathbf{x}-\mathbf{y}) \hat{\psi}^{\dagger}(\mathbf{y}, t) \hat{\psi}(\mathbf{y}, t) \hat{\psi}(\mathbf{x}, t)
$$

and

$$
\left(-i \frac{\partial}{\partial t^{\prime}}+\frac{\nabla_{\mathbf{x}}^{2}}{2 m}\right) \hat{\psi}^{\dagger}\left(\mathbf{x}, t^{\prime}\right)=\hat{\psi}^{\dagger}\left(\mathbf{x}, t^{\prime}\right) \int \mathrm{d}^{d} \mathbf{y} V(\mathbf{x}-\mathbf{y}) \hat{\psi}\left(\mathbf{y}, t^{\prime}\right) \hat{\psi}\left(\mathbf{y}, t^{\prime}\right) .
$$

Second The multiplication of Eq. (34) by $\hat{\psi}^{\dagger}(\mathbf{x}, t)$ on the leftTand the multiplication of Eq. (35) by $\hat{\psi}\left(\mathbf{x}, t^{\prime}\right)$ on the right can be combined to form

$$
\begin{aligned}
& \quad \frac{1}{4} \int \mathrm{d}^{d} \mathbf{x}\left[\left(i \frac{\partial}{\partial t}-i \frac{\partial}{\partial t^{\prime}}\right) \hat{\psi}^{\dagger}\left(\mathbf{x}, t^{\prime}\right) \hat{\psi}(\mathbf{x}, t)\right]_{t^{\prime}=t}=\frac{1}{4} \int \mathrm{d}^{d} \mathbf{x}\left[\left(-\frac{\nabla_{\mathbf{x}}^{2}}{2 m}-\frac{\nabla_{\mathbf{y}}^{2}}{2 m}\right) \hat{\psi}^{\dagger}(\mathbf{y}, t) \hat{\psi}(\mathbf{x}, t)\right]_{\mathbf{y}=\mathbf{x}} \\
& +\frac{1}{2} \int \mathrm{d}^{d} \mathbf{x} \mathrm{d}^{d} \mathbf{y} \hat{\psi}^{\dagger}(\mathbf{x}, t) \hat{\psi}^{\dagger}(\mathbf{y}, t) V(\mathbf{x}-\mathbf{y}) \hat{\psi}(\mathbf{y}, t) \hat{\psi}(\mathbf{x}, t) .
\end{aligned}
$$

Finally when

$$
\frac{1}{4} \int \mathrm{d}^{d} \mathbf{x}\left[\left(-\frac{\nabla_{\mathbf{x}}^{2}}{2 m}-\frac{\nabla_{\mathbf{y}}^{2}}{2 m}\right) \hat{\psi}^{\dagger}(\mathbf{y}, t) \hat{\psi}(\mathbf{x}, t)\right]_{\mathbf{y}=\mathbf{x}}
$$

is added to both sides of Eq. (36) and the statistical average (19) is takenTit follows that

$$
\begin{aligned}
\langle\hat{H}\rangle_{1} & =\frac{1}{4} \int \mathrm{d}^{d} \mathbf{x}\left[\left(i \frac{\partial}{\partial t}-i \frac{\partial}{\partial t^{\prime}}+\frac{\nabla_{\mathbf{x}} \cdot \nabla_{\mathbf{y}}}{m}\right)\left\langle\hat{\psi}^{\dagger}\left(\mathbf{y}, t^{\prime}\right) \hat{\psi}(\mathbf{x}, t)\right\rangle_{1}\right]_{\mathbf{y}=\mathbf{x}, t^{\prime}=t} \\
& = \pm \frac{i}{4} \int \mathrm{d}^{d} \mathbf{x}\left[\left(i \frac{\partial}{\partial t}-i \frac{\partial}{\partial t^{\prime}}+\frac{\nabla_{\mathbf{x}} \cdot \nabla_{\mathbf{y}}}{m}\right) G_{1<}\left(\mathbf{x}, t, \mathbf{y}, t^{\prime} ; \beta, \mu\right)\right]_{\mathbf{y}=\mathbf{x}, t^{\prime}=t} .
\end{aligned}
$$




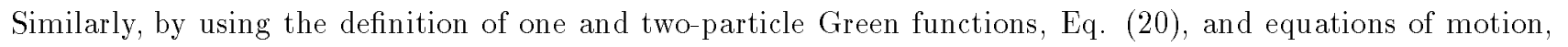
Eqs. (34) and (35) Гit can be verified that

$$
\begin{aligned}
& \left(i \frac{\partial}{\partial t_{1}}+\frac{\nabla_{\mathbf{x}}^{2}}{2 m}\right) G_{1}^{(1)}\left(\mathbf{x}_{1}, t_{1}, \mathbf{y}_{1}, t_{1}^{\prime} ; \beta ; \mu\right)=\delta\left(t_{1}-t_{1}^{\prime}\right) \delta(\mathbf{x}-\mathbf{y}) \\
& \pm\left. i \int \mathrm{d}^{d} \mathbf{y} V(\mathbf{x}-\mathbf{y}) G_{1}^{(2)}\left(\mathbf{x}_{1}, t_{1}, \mathbf{x}_{2}, t_{2}, \mathbf{y}_{1}, t_{1}^{\prime}, \mathbf{y}_{2}, t_{2}^{+} ; \beta ; \mu\right)\right|_{t_{2}=t_{1}} .
\end{aligned}
$$

The notation $t_{2}^{\prime+}$ is used to represent $t_{2}^{\prime}+\epsilon$ where $\epsilon$ is an infinitesimal positive number. Similar equations can be obtained for others $n$-particle Green functions $\Gamma$ giving a hierarchical structure.

\section{B.Generalized Green functions}

By taking into account the previous discussionsTa natural way to define a generalized $n$-particle Green function

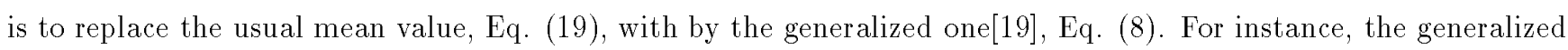
one-particle Green function becomes

$$
G_{q}^{\left(1^{\prime}\right)}\left(\mathbf{x}, t, \mathbf{y}, t^{\prime}\right)=\frac{1}{i}\left\langle\mathrm{~T}\left(\hat{\psi}(\mathbf{x}, t) \hat{\psi}^{\dagger}\left(\mathbf{y}, t^{\prime}\right)\right)\right\rangle_{q} .
$$

HoweverTas it is demonstrated in Sec. IVTthis Green function do not obey the usual equation of motion. In order

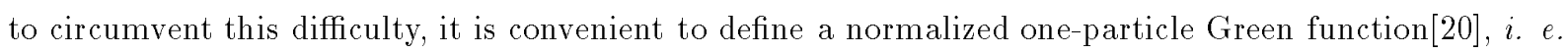

$$
G_{q}^{(1)}\left(\mathbf{x}, t, \mathbf{y}, t^{\prime}\right)=\frac{G_{q}^{\left(1^{\prime}\right)}\left(\mathbf{x}, t, \mathbf{y}, t^{\prime}\right)}{\langle\mathbf{1}\rangle_{q}}=\frac{1}{i\langle\mathbf{1}\rangle_{q}}\left\langle\mathrm{~T}\left(\hat{\psi}(\mathbf{x}, t) \hat{\psi}^{\dagger}\left(\mathbf{y}, t^{\prime}\right)\right)\right\rangle_{q}
$$

where $\langle\mathbf{1}\rangle_{q}=\operatorname{Tr} \hat{\rho}^{q}=1+(1-q) S_{q}$. A further definition of Green functions is discussed in Sec. VI.

As in the case of the generalized partition function (see Eq. (10)) The above one-particle Green function can be obtained from the usual one by using an integral representation. Indeed by using the definition of the usual Green

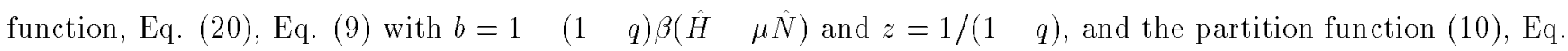
(41) can be written as

$$
G_{q}^{(1)}\left(\mathbf{x}, t, \mathbf{y}, t^{\prime} ; \beta, \mu\right)=\int_{C} \mathrm{~d} u K_{q}^{(2)}(u) Z_{1}(-\beta u(1-q), \mu) G_{1}^{(1)}\left(\mathbf{x}, t, \mathbf{y}, t^{\prime} ;-\beta u(1-q), \mu\right),
$$

with

$$
K_{q}^{(2)}(u)=i \frac{\Gamma(1 /(1-q))}{2 \pi\left(Z_{q}\right)^{q}\langle\mathbf{1}\rangle_{q}}(-u)^{-1 /(1-q)} \exp (-u) .
$$

Of course $\Gamma\langle\mathbf{1}\rangle_{q}$ can be expressed employing an integral representation. In this case $\Gamma$ it must be chosen $b=1-(1-$ q) $\beta(\hat{H}-\mu \hat{N})$ and $z=1 /(1-q)$.

In a similar way $\mathrm{Tthe}$ correlation functions $\Gamma G_{q}>$ for $t>t^{\prime}$ and $G_{q<}$ for $t<t^{\prime} \Gamma$ can be introduced as follows

$$
\begin{aligned}
G_{q>}\left(\mathbf{x}, t, \mathbf{y}, t^{\prime} ; \beta, \mu\right) & =\frac{1}{\langle\mathbf{1}\rangle_{q}}\left\langle\hat{\psi}(\mathbf{x}, t) \hat{\psi}^{\dagger}\left(\mathbf{y}, t^{\prime}\right)\right\rangle_{q} \\
& =\int_{C} \mathrm{~d} u K_{q}^{(2)}(u) Z_{1}(-\beta u(1-q), \mu) G_{1>}\left(\mathbf{x}, t, \mathbf{y}, t^{\prime} ;-\beta u(1-q), \mu\right)
\end{aligned}
$$

and

$$
\begin{aligned}
G_{q<}\left(\mathbf{x}, t, \mathbf{y}, t^{\prime} ; \beta, \mu\right) & =\frac{ \pm}{\langle\mathbf{1}\rangle_{q}}\left\langle\hat{\psi}^{\dagger}\left(\mathbf{y}, t^{\prime}\right) \hat{\psi}(\mathbf{x}, t)\right\rangle_{q} \\
& =\int_{C} \mathrm{~d} u K_{q}^{(2)}(u) Z_{1}(-\beta u(1-q), \mu) G_{1<}\left(\mathbf{x}, t, \mathbf{y}, t^{\prime} ;-\beta u(1-q), \mu\right)
\end{aligned}
$$

From Eqs. (44) and (45) it follows that the sum rule (28) is q invariant. In factГEqs. (44) and (30) leads to 


$$
\begin{gathered}
G_{q>}(\mathbf{p}, \omega ; \beta, \mu)=\int_{C} \mathrm{~d} u K_{q}^{(2)}(u) Z_{1}(-\beta u(1-q), \mu) G_{1>}(\mathbf{p}, \omega ;-\beta u(1-q), \mu) \\
=\int_{C} \mathrm{~d} u K_{q}^{(2)}(u)[1 \pm f(\omega ;-\beta u(1-q), \mu)] Z_{1}(-\beta u(1-q), \mu) A_{1}(\mathbf{p}, \omega ;-\beta u(1-q), \mu)
\end{gathered}
$$

and similarly

$$
\begin{gathered}
G_{q<}(\mathbf{p}, \omega ; \beta, \mu)=\int_{C} \mathrm{~d} u K_{q}^{(2)}(u) Z_{1}(-\beta u(1-q), \mu) G_{1<}(\mathbf{p}, \omega ;-\beta u(1-q), \mu) \\
=\int_{C} \mathrm{~d} u K_{q}^{(2)}(u) f(\omega ;-\beta u(1-q), \mu) Z_{1}(-\beta u(1-q), \mu) A_{1}(\mathbf{p}, \omega ;-\beta u(1-q), \mu),
\end{gathered}
$$

thus

$$
\begin{aligned}
& \int_{\infty}^{\infty} \frac{\mathrm{d} \omega}{2 \pi}\left[G_{q>}(\mathbf{p}, \omega ; \beta, \mu) \mp G_{q<}(\mathbf{p}, \omega ; \beta, \mu)\right] \\
= & \int_{C} \mathrm{~d} u K_{q}^{(2)}(u) Z_{1}(-\beta u(1-q), \mu) \int \frac{\mathrm{d} \omega}{2 \pi} A_{1}(\mathbf{p}, \omega ;-\beta u(1-q), \mu)=1 .
\end{aligned}
$$

Analogously to the usual case $\Gamma$ the physical contents of the theory can be obtained from the Green functions. This is exactly the case of $q$ expectation of the density of particles and Hamiltonian. Indeed from the usual definition of the expectation of the density of particlesTEq. (33) Tthe definition of the $q$ expectation of the density

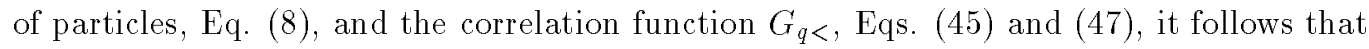

$$
\langle\hat{n}\rangle_{q}= \pm \int_{C} \mathrm{~d} u K_{q}^{(2)}(u) \int_{-\infty}^{\infty} \frac{\mathrm{d} \omega}{2 \pi} \int \frac{\mathrm{d}^{d} \mathbf{p}}{(2 \pi)^{d}} \frac{Z_{1}(-\beta(1-q) u, \mu) A_{1}(\mathbf{p}, \omega ; \beta, \mu)}{\exp (-\beta(1-q) u(\omega-\mu)) \mp 1} .
$$

Thus the chemical potential can be determined in terms of the $q$ mean value by using Eq. (49). Following the

calculations developed to obtain Eq. (38) one concludes analogously that $\langle\hat{H}\rangle_{q}$ can be obtained from $G_{q<} \Gamma b e c a u s e$ the calculation in both cases $(q=1$ and $q \neq 1)$ is the sameTthus

$$
\begin{aligned}
\langle\hat{H}\rangle_{q} & =\frac{1}{4} \int \mathrm{d}^{d} \mathbf{x}\left[\left(i \frac{\partial}{\partial t}-i \frac{\partial}{\partial t^{\prime}}+\frac{\nabla \mathbf{x} \cdot \nabla \mathbf{y}}{m}\right)\left\langle\hat{\psi}^{\dagger}(\mathbf{y}, t) \hat{\psi}(\mathbf{x}, t)\right\rangle_{q}\right]_{\mathbf{y}=\mathbf{x}, t^{\prime}=t} \\
& = \pm \frac{1}{4}\langle\mathbf{1}\rangle_{q} \int \mathrm{d}^{d} \mathbf{x}\left[\left(i \frac{\partial}{\partial t}-i \frac{\partial}{\partial t^{\prime}}+\frac{\nabla_{\mathbf{x}} \cdot \nabla_{\mathbf{y}}}{m}\right) G_{q<}\left(\mathbf{x}, t, \mathbf{x}, t^{\prime} ; \beta, \mu\right)\right]_{\mathbf{y}=\mathbf{x}, t^{\prime}=t} .
\end{aligned}
$$

\section{Hartree and Hartree-Fock Approximations}

The generalized $n$-particle Green function is defined as

$$
\begin{aligned}
& G_{q}^{(n)}\left(\mathbf{x}_{1}, t_{1}, \ldots, \mathbf{x}_{n}, t_{n}, \mathbf{y}_{1}, t_{1}^{\prime}, \ldots, \mathbf{y}_{n}, t_{n}^{\prime} ; \beta ; \mu\right) \\
= & \frac{1}{i^{n}\langle\mathbf{1}\rangle_{q}}\left\langle\mathrm{~T}\left(\hat{\psi}\left(\mathbf{x}_{1}, t_{1}\right) \ldots \hat{\psi}\left(\mathbf{x}_{n}, t_{n}\right) \hat{\psi}^{\dagger}\left(\mathbf{y}_{1}, t_{1}^{\prime}\right) \ldots \hat{\psi}^{\dagger}\left(\mathbf{y}_{n}, t_{n}^{\prime}\right)\right)\right\rangle_{q} \\
= & \int_{C} \mathrm{~d} u K_{q}^{(2)}(u) Z_{1}(-\beta u(1-q), \mu) G_{1}^{(n)}\left(\mathbf{x}_{1}, t_{1}, \ldots, \mathbf{x}_{n}, t_{n}, \mathbf{y}_{1}, t_{1}^{\prime}, \ldots, \mathbf{y}_{n}, t_{n}^{\prime} ;-\beta u(1-q) ; \mu\right)
\end{aligned}
$$

As in the usual case $\Gamma$ a set of equations for these Green functions can be obtained. MoreoverT the form of these equations is $q$ invariant. For instance $\Gamma$ the motion equation for $G_{q}^{(1)}$ is

$$
\begin{aligned}
& \left(i \frac{\partial}{\partial t_{1}}+\frac{\nabla_{\mathbf{x}}^{2}}{2 m}\right) G_{q}^{(1)}\left(\mathbf{x}_{1}, t_{1}, \mathbf{y}_{1}, t_{1}^{\prime} ; \beta ; \mu\right)=\delta\left(t_{1}-t_{1}^{\prime}\right) \delta(\mathbf{x}-\mathbf{y}) \\
& \pm\left. i \int \mathrm{d}^{d} \mathbf{y} V(\mathbf{x}-\mathbf{y}) G_{q}^{(2)}\left(\mathbf{x}_{1}, t_{1}, \mathbf{x}_{2}, t_{2}, \mathbf{y}_{1}, t_{1}^{\prime}, \mathbf{y}_{2}, t_{2}^{\prime+} ; \beta ; \mu\right)\right|_{t_{2}=t_{1}}
\end{aligned}
$$


In fact Tproceeding similarly to the usual case it is possible to verify that the form of Eqs. (39) and (52) are the same.

In generalTto solve the equations of motion is a formidable task. Of course Tthis is not different in the nonextensive Tsallis statistical mechanics. Following the usual caseTtwo approximated methods are usually employed. The first one is the Hartree approximation. It is supposed $\Gamma$ in this simple approximation $\Gamma$ that $G_{1}^{(2)}$ becomes the product $G_{1}^{(1)} G_{1}^{(1)}$. In this wayTa natural generalization of the Hartree approximations is

$$
G_{q}^{(2)}\left(\mathbf{x}_{1}, t_{1}, \mathbf{x}_{2}, t_{2}, \mathbf{y}_{1}, t_{1}^{\prime}, \mathbf{y}_{2}, t_{2}^{\prime} ; \beta ; \mu\right)=G_{q}^{(1)}\left(\mathbf{x}_{1}, t_{1}, \mathbf{y}_{1}, t_{1}^{\prime} ; \beta ; \mu\right) G_{q}^{(1)}\left(\mathbf{x}_{2}, t_{2}, \mathbf{y}_{2}, t_{2}^{\prime} ; \beta ; \mu\right)
$$

In this approximationTit was not considered the exclusion principle. When this new property is take into account $\Gamma$ Eq. (53) should be substituted by

$$
\begin{aligned}
G_{q}^{(2)}\left(\mathbf{x}_{1}, t_{1}, \mathbf{x}_{2}, t_{2}, \mathbf{y}_{1}, t_{1}^{\prime}, \mathbf{y}_{2}, t_{2}^{\prime} ; \beta ; \mu\right) & =G_{q}^{(1)}\left(\mathbf{x}_{1}, t_{1}, \mathbf{y}_{1}, t_{1}^{\prime} ; \beta ; \mu\right) G_{q}^{(1)}\left(\mathbf{x}_{2}, t_{2}, \mathbf{y}_{2}, t_{2}^{\prime} ; \beta ; \mu\right) \\
& \pm G_{q}^{(1)}\left(\mathbf{x}_{1}, t_{1}, \mathbf{y}_{2}, t_{2}^{\prime} ; \beta ; \mu\right) G_{q}^{(1)}\left(\mathbf{x}_{2}, t_{2}, \mathbf{y}_{1}, t_{1}^{\prime} ; \beta ; \mu\right)
\end{aligned}
$$

This is the generalized Hartree-Fock approximation. Further consequences of these approximations are presented in Ref. [20].

\section{Path integral formulation}

As discussed in Sec. IITthe generalized partition function $\Gamma Z_{q} \Gamma$ can be expressed in terms of $Z_{1}$ (see Eq. (10)). This fact can be employed to write $Z_{q}$ in terms of a path integral[30]. In fact Tby using the path integral representation of the usual partition function (see $\Gamma$ for instanceTRef. [31] ch. III) $\Gamma$

$$
Z_{1}=\int \cdots \int \mathcal{D} \bar{\psi} \mathcal{D} \psi \exp \left(-\int_{0}^{\beta} \mathrm{d} \tilde{t} \int \mathrm{d}^{d} \mathrm{x}(\bar{\psi} \dot{\psi}+H(\bar{\psi}, \psi))\right)
$$

and the integral representation (10) Tit is immediate to verify that

$$
Z_{q}=\int_{C} \mathrm{~d} u K_{q}^{(1)}(u) \int \ldots \int \mathcal{D} \bar{\psi} \mathcal{D} \psi \exp \left(-\int_{0}^{\beta^{*}} \mathrm{~d} \tilde{t} \int \mathrm{d}^{d} \mathbf{x}(\bar{\psi} \dot{\psi}+H(\bar{\psi}, \psi))\right)
$$

where $\beta^{*}=(1-q)(-u) \beta$. The functional generator of the Green functions $\Gamma \mathcal{Z}_{q}(\bar{J}, J)$ Tcan be obtained in a similar way. However $\Gamma$ it is necessary to employ the kernel $K_{q}^{(2)}$ instead of $K_{q}^{(1)} \Gamma i$. e. $\Gamma$

$$
\begin{aligned}
& \mathcal{Z}_{q}(\bar{J}, J)= \\
& \int_{C} \mathrm{~d} u K_{q}^{(2)}(u) \int \ldots \int \mathcal{D} \bar{\psi} \mathcal{D} \psi \exp \left(-\int_{0}^{\beta^{*}} \mathrm{~d} \tilde{t} \int \mathrm{d}^{d} \mathbf{x}(\bar{\psi} \psi+H(\bar{\psi}, \psi)-\bar{J} \psi-\bar{\psi} J)\right) .
\end{aligned}
$$

FinallyTthe $n$-particle Green function $\Gamma \tilde{G}_{q}^{(n)}\left(\mathbf{x}_{1}, \ldots, \mathbf{x}_{n}, \mathbf{y}_{1}, \ldots, \mathbf{y}_{n}\right)$ Tis obtained by taking functional derivatives with relation to the sources $\bar{J}$ and $J$. Of course Tthese temperature Green functions are time-independent.

\section{Green functions and normalized statistics}

RecentlyTit was considered a new kind of generalized mean value[32] Tsee also Ref.[21]

$$
\langle\langle A\rangle\rangle_{q}=\frac{\operatorname{Tr} \hat{\rho}^{q} \hat{A}}{\operatorname{Tr} \hat{\rho}^{q}}
$$


By using this new definition Tthe grand-canonical distribution can be obtained maximizing the Tsallis entropy (see Eq. (1)) subject to the constraints $\operatorname{Tr} \hat{\rho}=1 \Gamma$

$$
\frac{\operatorname{Tr} \hat{\rho}^{q} \hat{H}}{\operatorname{Tr} \hat{\rho}^{q}}=U_{q}^{(2)}
$$

and

$$
\frac{\operatorname{Tr} \hat{\rho}^{q} \hat{N}}{\operatorname{Tr} \hat{\rho}^{q}}=N_{q}^{(2)} .
$$

In this wayTthe new grand-canonical distribution becomes

$$
\hat{\rho}=\left\{1-(1-q) \beta\left[\hat{H}-U_{q}^{(2)}-\mu\left(\hat{N}-N_{2}^{(2)}\right)\right] / \operatorname{Tr} \hat{\rho}^{q}\right\}^{1 /(1-q)} / Z_{q}^{(2)},
$$

where

$$
Z_{q}^{(2)}=\operatorname{Tr}\left\{1-(1-q) \beta\left[\hat{H}-U_{q}^{(2)}-\mu\left(\hat{N}-N_{2}^{(2)}\right)\right] / \operatorname{Tr} \hat{\rho}^{q}\right\}^{1 /(1-q)} .
$$

The $q$-expectation value defined by Eq. (58) leads to $\langle\langle\mathbf{1}\rangle\rangle_{q}=1$ instead of $\langle\mathbf{1}\rangle_{q} \neq 1$. Furthermore 5 the grandcanonical distribution does not depend of the choice on the origin of the ground state energy contrary to the distribution given by Eq. (5). These properties basically indicate that this new formulation is the correct one for the nonextensive statistical mechanics[33]. HoweverTthe calculations based on the distribution (61) are difficult to be performed because the density matrix depends explicitly on $U_{q}^{(2)}$ and $N_{q}^{(2)}$. More preciselyГthis fact indicates that calculations in this new formulation must be performed in a self-consistent way. On the other hand $\Gamma$ the formal development discussed in the previous sections can be extended directly for this new case. For instance $\Gamma$ the $n$-particle Green function $\Gamma G_{q}^{(n)(2)}$ Tbased on the new formulation can be defined in the following way:

$$
\begin{aligned}
& G_{q}^{(n)(2)}\left(\mathbf{x}_{1}, t_{1}, \ldots, \mathbf{x}_{n}, t_{n}, \mathbf{y}_{1}, t_{1}^{\prime}, \ldots, \mathbf{y}_{n}, t_{n}^{\prime}\right)= \\
& \frac{1}{i^{n}}\left\langle\left\langle\mathrm{~T}\left(\hat{\psi}\left(\mathbf{x}_{1}, t_{1}\right) \ldots \hat{\psi}\left(\mathbf{x}_{n}, t_{n}\right) \hat{\psi}^{\dagger}\left(\mathbf{y}_{1}, t_{1}^{\prime}\right) \ldots \hat{\psi}^{\dagger}\left(\mathbf{y}_{n}, t_{n}^{\prime}\right)\right)\right\rangle\right\rangle_{q} .
\end{aligned}
$$

Other examples are the motion equations for Green functions $\Gamma$ for instance $\Gamma G_{q}^{(1)}$ and $G_{q}^{(2)}$ must be respectively

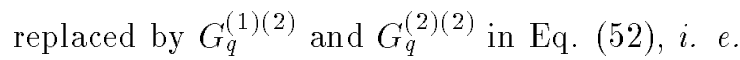

$$
\begin{aligned}
& \left(i \frac{\partial}{\partial t_{1}}+\frac{\nabla_{\mathbf{x}}^{2}}{2 m}\right) G_{q}^{(1)(2)}\left(\mathbf{x}_{1}, t_{1}, \mathbf{y}_{1}, t_{1}^{\prime} ; \beta ; \mu\right)=\delta\left(t_{1}-t_{1}^{\prime}\right) \delta(\mathbf{x}-\mathbf{y}) \\
& \pm\left. i \int \mathrm{d}^{d} \mathbf{y} V(\mathbf{x}-\mathbf{y}) G_{q}^{(2)(2)}\left(\mathbf{x}_{1}, t_{1}, \mathbf{x}_{2}, t_{2}, \mathbf{y}_{1}, t_{1}^{\prime}, \mathbf{y}_{2}, t_{2}^{++} ; \beta ; \mu\right)\right|_{t_{2}=t_{1}}
\end{aligned}
$$

The application of these developments to study a simple many-body system Tthe quantum ideal gas Tleads to a set of coupled equations. In generalTthe solutions of these equations must be performed numerically.

\section{Non-Gaussian integration}

A very useful tool in the context of path integrals is based on Gaussian onesTi. e. in the generating function[34]

$$
\begin{aligned}
\mathcal{Z}_{1, \sigma}\left(G, \bar{J}, \bar{x}^{(0)}\right) & =\int \mathcal{D} x \exp \left(-\frac{\sigma}{2}\left(\bar{x}-\bar{x}^{(0)}\right) G^{-1}\left(\bar{x}-\bar{x}^{(0)}\right)+\bar{J} \cdot \bar{x}\right) \\
& =\left(\frac{2 \pi}{\sigma}\right)^{N / 2}(\operatorname{det} G)^{1 / 2} \exp \left(\frac{\bar{J} G \bar{J}}{2 \sigma}+\bar{J} \cdot \bar{x}^{(0)}\right) .
\end{aligned}
$$

Here $\mathcal{D} x=\prod_{i=1}^{N} \mathrm{~d} x_{i} \Gamma \bar{J} \cdot \bar{x}=\sum_{i=1}^{N} J_{i} x_{i}$ and $\bar{x} G^{-1} \bar{x}=\sum_{i, j=1}^{N} x_{i} G_{i j}^{-1} x_{j}$ Tand $N$ is the space dimension. Furthermore $\Gamma$ the parameter $\sigma$ was introduced for future convenience. This section is dedicated to the generalization of the Gaussian integral (65) based on Tsallis statistical weight[35]. 
As it was emphasized bellow Eq. $(7) \Gamma[1+(1-q) x]^{1 /(1-q)}$ is a natural generalization of the exponential function in the present context. ThusT the function

$$
f_{q}^{(1)}(G, x)=\left[1-\frac{(1-q)}{2 G}\left(x-x^{(0)}\right)^{2}\right]^{1 /(1-q)}
$$

can be called $q$-GaussianTbecause it is reduced to $\exp \left(-\left(x-x^{(0)}\right)^{2} /(2 G)\right)$ in the limit $q \rightarrow 1$. In this wayTthe $N$-dimensional q-Gaussian becomes

$$
f_{q}^{(N)}(G, \bar{x})=\left[1-\frac{1-q}{2}\left(\bar{x}-\bar{x}^{(0)}\right) G^{-1}\left(\bar{x}-\bar{x}^{(0)}\right)\right]^{1 /(1-q)} .
$$

By using $f_{q}^{(N)}$ Tit is possible to generalize the generating function (65) in the following way

$$
\mathcal{Z}_{q, \alpha}\left(G, \bar{J}, \bar{x}^{(0)}\right) \equiv \int \mathcal{D} x\left(f_{q}^{(N)}(G, \bar{x})\right)^{\alpha} \exp (\bar{J} \cdot \bar{x})
$$

since

$$
P_{q, \alpha}\left(x_{j_{1}} x_{j_{2}} \ldots x_{j_{s}}\right) \equiv \int \mathcal{D} x x_{j_{1}} x_{j_{2}} \ldots x_{j_{s}}\left(f_{q}^{(N)}(G, \bar{x})\right)^{\alpha}=\left.\frac{\partial^{s} \mathcal{Z}_{q, \alpha}\left(G, \bar{J}, \bar{x}^{(0)}\right)}{\partial J_{j_{1}} \partial J_{j_{2}} \ldots \partial J_{j_{s}}}\right|_{\bar{J}=0} .
$$

The parameter $\alpha$ was introduced because $\alpha \neq 1$ occurs in several situations $\Gamma$ for instance $\Gamma$ when it is necessary to calculate $\langle A\rangle_{q}=\operatorname{Tr} \hat{\rho}^{q} \hat{A}$ in classical systems as the ideal gas.

In general $\Gamma$ the integral (69) is divergent for $q \geq 1+2 \alpha /(N+s)$. In fact $\Gamma$ since $\mathcal{D} x \propto|\bar{x}|^{N-1} \mathrm{~d}|\bar{x}|$ and $\left(f_{q}^{(N)}\right)^{\alpha} \propto|\bar{x}|^{2 \alpha /(1-q)}$ for large $|\bar{x}|$ in the representative case $G_{i j}^{-1} \propto \delta_{i j} \Gamma$ Eq. (69) contains an integral proportional to $\int \mathrm{d}|\bar{x}||\bar{x}|^{N-1+s-2 \alpha /(q-1)}$ for a sufficiently large $|\bar{x}|$. On the other handTfollowing the general discussion presented in Sec. IIT $f_{q}^{(N)}$ contains a cut off for $q<1$. Consequently $\mathrm{Sthe}$ integral on the left side of Eq. (69) is convergent for arbitrary $N$ when $q<1$. The present discussion is restrict to the convergent case $\Gamma i . e . \Gamma$ the $q<1$ one.

Employing the Lenzi representation (see Eq. (13)) with $a=1 \Gamma b=1-(1-q)\left(\bar{x}-\bar{x}^{(0)}\right) G^{-1}\left(\bar{x}-\bar{x}^{(0)}\right) / 2$ and $z=\alpha /(1-q)+1$ in Eq. (67) Tit follows that

$$
\left(f_{q}^{(N)}(G, \bar{x})\right)^{\alpha}=\int_{-\infty}^{\infty} \mathrm{d} t K_{q}^{(3)}(t) \exp \left(-\frac{\sigma}{2}\left(\bar{x}-\bar{x}^{(0)}\right) G^{-1}\left(\bar{x}-\bar{x}^{(0)}\right)\right)
$$

with

$$
K_{q}^{(3)}(t)=\frac{\Gamma(\alpha /(1-q)+1) \exp (1+i t)}{2 \pi(1+i t)^{\alpha /(1-q)+1}}
$$

and $\sigma=(1-q)(1+i t)$. Thus Eq. (68) becomes

$$
\mathcal{Z}_{q, \alpha}\left(G, \bar{J}, \bar{x}^{(0)}\right)=\int_{-\infty}^{\infty} \mathrm{d} t K_{q}^{(3)}(t) \mathcal{Z}_{1, \sigma}\left(\bar{J}, \bar{x}^{(0)}\right)
$$

The final expression for the generating function can be obtained by expanding the last exponential in power series and by using Eq. (13) again with $a=b=1$ to calculate each term of the integral. This calculation leads to

$$
\begin{aligned}
\mathcal{Z}_{q, \alpha}\left(G, \bar{J}, \bar{x}^{(0)}\right) & =\mathcal{Z}_{q, \alpha}(G, 0,0) \exp \left(\bar{J} \cdot \bar{x}^{(0)}\right) \sum_{n=0}^{\infty} \frac{\Gamma(\alpha /(1-q)+1+N / 2)}{n ! \Gamma(\alpha /(1-q)+1+N / 2+n)}\left(\frac{\bar{J} G \bar{J}}{2(1-q)}\right)^{n} \\
& =\mathcal{Z}_{q, \alpha}(G, 0,0) \exp \left(\bar{J} \cdot \bar{x}^{(0)}\right) \Gamma(\mu+1)\left(\frac{\bar{J} G \bar{J}}{2(1-q)}\right)^{-\mu / 2} I_{\mu}\left(\left(\frac{2 \bar{J} G \bar{J}}{1-q}\right)^{1 / 2}\right)
\end{aligned}
$$

where

$$
\mathcal{Z}_{q, \alpha}(G, 0,0)=\left(\frac{2 \pi}{1-q}\right)^{N / 2} \frac{\Gamma(\alpha /(1-q)+1)}{\Gamma(\alpha /(1-q)+1+N / 2)}(\operatorname{det} G)^{1 / 2}
$$


$I_{\mu}(x)=\sum_{n=0}^{\infty}(x / 2)^{2 n+\mu} /[n ! \Gamma(n+1+\mu)]$ is the modified Bessel function of first kind $\Gamma$ and $\mu=\alpha /(1-q)+N / 2$.

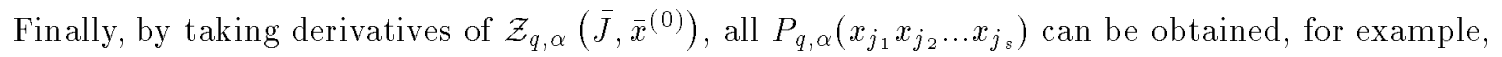

$$
\begin{aligned}
P_{q, \alpha}(1) & =\mathcal{Z}_{q, \alpha}(G, 0,0) \\
P_{q, \alpha}\left(x_{j_{1}}\right) & =\mathcal{Z}_{q, \alpha}(G, 0,0) x_{j_{1}}^{(0)} \\
P_{q, \alpha}\left(x_{j_{1}} x_{j_{2}}\right) & =\mathcal{Z}_{q, \alpha}(G, 0,0)\left(x_{j_{1}}^{(0)} x_{j_{2}}^{(0)}+\frac{G_{j_{1} j_{2}}}{\alpha+(1-q)(N / 2+1)}\right) .
\end{aligned}
$$

A similar procedure can be employed when $1<q<1+2 \alpha /(N+\alpha)$ rbut it is necessary to replace consistently the Lenzi representation with the Hilhorst one.

A correlation function of the product $x_{j_{1}} x_{j_{2}} \ldots x_{j_{s}} \Gamma\left\langle x_{j_{1}} x_{j_{2}} \ldots x_{j_{s}}\right\rangle_{q, \alpha}$ can be defined in terms of the above results by taking the relation

$$
\left\langle x_{j_{1}} x_{j_{2}} \ldots x_{j_{s}}\right\rangle_{q, \alpha} \equiv P_{q, \alpha}\left(x_{j_{1}} x_{j_{2}} \ldots x_{j_{s}}\right) / P_{q, \alpha}(1) .
$$

From this definition with $\bar{x}^{(0)}=0$ it follows the relation between $\left\langle x_{j_{1}} x_{j_{2}} \ldots x_{j_{2 n}}\right\rangle_{q, \alpha}$ and $\left\langle x_{j_{1}} x_{j_{2}}\right\rangle_{q, \alpha}=G_{j_{1} j_{2}} /[\alpha+$ $(1-q)(N / 2+1)]$ Twhere $n$ is an integer greater than one. A direct calculation leads to

$$
\left\langle x_{j_{1}} x_{j_{2}} \ldots x_{j_{2 n}}\right\rangle_{q, \alpha}=\prod_{k=1}^{n} \frac{\alpha+(1-q)(N / 2+1)}{\alpha+(1-q)(N / 2+k)} \sum_{\text {perm }}\left\langle x_{j_{P_{1}}} x_{j_{P_{2}}}\right\rangle_{q, \alpha} \ldots\left\langle x_{j_{P_{2 n}-1}} x_{j_{P_{2 n}}}\right\rangle_{q, \alpha},
$$

where $P_{k}$ represents a permutation and $\sum_{\text {perm }}$ indicates a sum over all permutations without repeating $\left\langle x_{j_{1}} x_{j_{P_{2}}}\right\rangle_{q, \alpha}$ Tsince $\left\langle x_{j_{P_{k}}} x_{j_{P_{n}}}\right\rangle_{q, \alpha}=\left\langle x_{j_{P_{n}}} x_{j_{P_{k}}}\right\rangle_{q, \alpha}$. The above result resembles the Wick's theorem (seeTfor instance $\Gamma$ Ref. [36]) $\Gamma$ and in particular the usual Wick's theorem is recovered in the limit $q \rightarrow 1$ and $N \rightarrow \infty$. For the general case $E q$. (77) formally differs from the usual one by a factor that decreases when $n$ increases. Moreover $\Gamma$ this difference from one increases when $q$ decreases and $N$ increases. In conclusion $\mathrm{Eq}$. (77) is a generalization of Wick's theorem based on $f_{q}^{(N)}$ for discrete systems.

Applications of the $N$-dimensional non-Gaussian integration are useful in several contexts. For instance $\Gamma$ in the study of the Tsallis statistical mechanics of the classical ideal gas by using the unnormalized formulation[37] or the normalized one[38] Tbecause the partition function is proportional to $\mathcal{Z}_{q, 1}(m \mathbf{1}, 0,0)$ (see Eq. (68)) with $\bar{x}=\left(\bar{p}_{1}, \bar{p}_{2}, \ldots\right) \Gamma$ where $\bar{p}_{i}$ is the momentum of the $i$-th particle. A second example is related with the variational methods $\Gamma$ in this case $f_{q}^{N}$ is the trial function. For example $\Gamma$ the ap- plication of this trial function to study the equation $E \phi=\left(-\hbar^{2} /(2 m) \partial^{2} / \partial x^{2}+\lambda x^{4}\right) \phi$ leads to a very good approximation of the ground state energy[35].

\section{Discussions and conclu- sions}

A detailed discussion about generalized Green functions was presented. The integral representations were important tools in this discussionT because they can be used to connect the usual case $\Gamma q=1 \Gamma$ and the generalized one $\Gamma q \neq 1$. This fact indicates that integral representations can be employed as guide to generalize and calculate many quantities related with the formal structure of the nonextensive Tsallis statistical mechanics. The generalized Gaussian integrals are other typical examples of this formal structure.

Since the usual Green functions and path integral are important tools in the study of extensive systems $\Gamma$ it is expected that the generalized Green functions and path integral formulation of nonextensive Tsallis statistical mechanics become useful in the discussion 
of nonextensive systems. In generalT the calculations based on generalized Green functions and path integral are more elaborated than the corresponding case with $q=1$. Furthermore $\Gamma$ the analysis is more difficult to be performed in the case of the normalized nonextensive Tsallis statistical mechanics than in the unnormalized oneTbecause coupled equations must be solved. In particularTgeneralized Green functions and path integral are not employed yet to analyze many-body systems other than the quantum ideal gas.

The generalized Gaussian integral presented in Sec. VII can be used in several contexts. Obvious applications of these integrals come from nonextensive Tsallis statistical mechanics of the classical ideal gas and of a set of harmonic oscillators. Of course Tperturbations of these systems can be analyzed too. Other applications can be based on situations where deviations from the Gaussian behavior is expected. A typical example is the ground state of anharmonic oscillators.

\section{Acknowledgements}

The author thanks E. K. Lenzi A. K. Rajagopal and C. Tsallis for helpful discussions.

\section{References}

[1] W.C. Saslaw, Gravitational physics of stellar and galactic systems (Cambridge University Press, Cambridge, 1985).

[2] J. Binney and S. Tremaine, Galactic dynamics (Princeton University Press, Princeton, 1987).

[3] H. Risken, The Fokker-Planck equation (SpringerVerlag, Berlin, 1984), p. 9.

[4] C. Tsallis, J. Stat. Phys. 52, 479 (1988).

[5] D. H. Zanette and P. A. Alemany, Phys. Rev. Lett. 75, 366 (1995); M. O. Cáceres and C. E. Budde, ibid. 77, 2589 (1996); D. H. Zanette and P. A. Alemany, ibid. 77, 2590 (1996).

[6] C. Tsallis, S. V. F. Levy, A. M. C. Souza, and R. Maynard, Phys. Rev. Lett. 75, 3589 (1995); 77, 5442(E) (1996).

[7] A. R. Plastino and A. Plastino, Physica A 222, 347 (1995); C. Tsallis and D. J. Bukman, Phys. Rev. E 54, R2197 (1996); L. Borland, ibid. 57, 6634 (1998).

[8] B. M. Boghosian, Phys. Rev. E 53, 4754 (1996).

[9] I. Koponen, Phys. Rev. E. 55, 7759 (1997).

[10] V. H. Hamity and D. E. Barraco, Phys. Rev. Lett. 76, 4664 (1996); D. F. Torres, H. Vucetich, and A. Plastino, ibid. 79, 1588 (1997).
[11] M. L. Lyra and C. Tsallis, Phys. Rev. Lett. 80, 53 (1998).

[12] C. Anteneodo and C. Tsallis, Phys. Rev. Lett. 80, 5313 (1998).

[13] See http://tsallis.cat.cbpf.br/biblio.htm for an updated bibliography on the subject.

[14] E. M. F. Curado and C. Tsallis, J. Phys. A: Math. Gen. 24, L69 (1991); 24, 3187(E); 25, 1019(E) (1992).

[15] A. Plastino and A. R. Plastino, Phys. Lett. A 226, 257 (1997); R. S. Mendes, Physica A 242, 299 (1997).

[16] A. K. Rajagopal, Phys. Rev. Lett. 76, 3469 (1996).

[17] E. K. Lenzi, L. C. Malacarne, and R. S. Mendes, Phys. Rev. Lett. 80, 218 (1998);

[18] R. S. Mendes, Kwok Sau Fa, J. N. Maki, and E. K. Lenzi, Perturbation expansion, Bogoliubov inequality and integral representations in nonextensive Tsallis statitics, Eur. Phys. J. B (to be published).

[19] A. K. Rajagopal, R. S. Mendes, and E. K. Lenzi, Phys. Rev. Lett. 80, 3907 (1998).

[20] E. K. Lenzi. R. S. Mendes, and A. K. Rajagopal, Phys. Rev. E 59, 1398 (1999).

[21] S. Abe, The thermal Green functions in nonextensive quantum statistical mechanics, preprint (1998).

[22] E. T. Wittaker and G. N. Watson, A Course of Modern Analysis, (Cambridge University Press, New York, 1973 ) ch. XII.

[23] C. Tsallis in New trends in magnetism, magnetic materials, and their applications, eds. J. L. Morán-López and J. M. Sanchez, (Penum Press, New York, 1994) p. 451.

[24] E. K. Lenzi, (unpublished) (1997).

[25] I. S. Gradshteyn and I. M. Ryzhik, Table of Integrals Series and Products (Academic Press, New York, 1965), p. $935,8.315-2$.

[26] E. K. Lenzi and R. S. Mendes, Phys. Lett. A 250, 270 (1998).

[27] D. Prato, Phys. Lett. A 203, 195 (1995).

[28] The notation employed here follows closely Kadanoff and Baym[29].

[29] L. P. Kadanoff and G. Baym, Quantum Statistical Mechanics, (W. A. Benjamin, Inc., New York, 1962).

[30] E. K. Lenzi, L. C. Malacarne and R. S. Mendes, Path integral and Bloch equation in nonextensive Tsallis statistics, preprint (1998).

[31] B. Sakita, Quantum Theory of Many-variable Systems and Fields (Word Scientific, Singapore, 1985).

[32] C. Tsallis, R. S. Mendes and A. R. Plastino, Physica A 261, 534 (1998).

[33] A detailed discussion of this question is presented in Ref. [32].

[34] R. Kubo, M. Toda and N. Hashitsume, Statistical Physics II, (Springer-Verlag, Berlin, 1985), p. 22-25. 
[35] R. S. Mendes, Kwok Sau Fa and E. K. Lenzi, Variational methods and non-Gaussian states based on Tsallis statistics, preprint (1998).

[36] D. J. Amit, Field Theory, the Renormalization Group, and Critical Phenomena (McGraw-Hill, New York, 1978), p. 54-55; see also Ref. [34].
[37] A. R. Plastino, A. Plastino and C. Tsallis, J. Phys. A: Math. Gen. 27, 5707 (1994).

[38] S. Abe, Thermodynamic limit and classical ideal gas in nonextensive statistical mechanics with normalized q-expection values, preprint (1998). 